\title{
Association of Major Depressive Disorder on Heart Failure With Reduced and Preserved Ejection Fraction: Analysis of National Readmission Database 2018
}

\author{
Harshith S. Thyagaturu ${ }^{1}$, Sittinun Thangjui ${ }^{1}$, Kashyap Shah ${ }^{2}$, Riddhima V. Naik ${ }^{1}$, Gayatri Bondi ${ }^{1}$ \\ 1. Internal Medicine, Bassett Healthcare Network, Cooperstown, USA 2. Internal Medicine, Saint Luke's University \\ Hospital, Bethlehem, USA
}

Corresponding author: Harshith S. Thyagaturu, harshihbk@gmail.com

\section{Abstract}

\section{Introduction}

The effect of major depressive disorder (MDD) on heart failure types is unclear. We aimed to assess the association of depression in heart failure with preserved ejection fraction (HFpEF) and heart failure with reduced ejection fraction (HFrEF) readmissions using the Nationwide Readmission Database (NRD) 2018.

\section{Methods}

We identified hospitalizations with a primary discharge diagnosis of HFrEF and HFpEF by appropriate ICD$10-\mathrm{CM}$ codes. We acquired mortality and readmission data with and without MDD at 30 days. We used multivariate logistic regression analysis to estimate the adjusted odds ratio (aOR).

\section{Results}

Among 102,997 patients admitted with heart failure as a primary diagnosis, $11 \%$ had MDD. We found a similar prevalence of HFpEF with MDD compared to HFrEF at $13.9 \%$ and 10\%, respectively. Both HFrEF and HFpEF patients with MDD had similar combined outcomes of 30-day mortality and rehospitalization compared to patients without MDD with aOR 0.94 (95\% CI: 0.85-1.04) and 0.93 (95\% CI: 0.81-1.07), respectively. Both types of HF with MDD were associated with lesser mortality.

\section{Conclusion}

MDD was associated with similar combined 30-day mortality and readmissions for both HFrEF and HFpEF. However, MDD was associated with decreased 30-day mortality in both groups of heart failure (HF) patients. Further studies with robust medications and treatment data are needed to verify the results of our study.

Review began 05/04/2021 Review ended 05/16/2021 Published 05/19/2021

\section{() Copyright 2021}

Thyagaturu et al. This is an open access article distributed under the terms of the Creative Commons Attribution License CC-BY 4.0., which permits unrestricted use, distribution, and reproduction in any medium, provided the original author and source are credited.
Categories: Cardiology, Internal Medicine, Psychiatry

Keywords: heart failure, cardiac failure, depression, major depressive disorder, readmission, mortality

\section{Introduction}

Depression has shown to be associated with worsening outcomes in heart failure (HF) patients since early 2000. A meta-analysis in 2006 showed a $22 \%$ prevalence of depression in HF patients. The prevalence of depression in this meta-analysis was higher in studies that used the questionnaires (e.g., Beck Depression Scale, Geriatric Depression Scale, etc.) to diagnose depression compared to clinician diagnosis. The depressive disorder was also associated with a greater than a two-fold risk of mortality and associated clinical events [1]. This result was similar to the prevalence studies done during 2007 to 2010 in the United States that showed nearly a two-fold higher risk of hospital admission and emergency department visits and a four-fold increase in mortality in HF patients with moderate to severe depression [2]. However, data from 2010 to 2014 from the Nationwide Readmission Database, which included approximately 60\% of hospitalized patients in the United States, showed only a modest increase in readmission rates in patients with depression after adjusting for other comorbidities [3]. Similarly, a Danish database from 1995 to 2014 showed a modest increase in mortality in heart failure patients with depression [4]. Given this significant risk of poor outcome in HF patients with depression, psychosocial intervention and pharmacological treatment were studied for depression in HF patients. Major antidepressant trials using sertraline in heart failure with reduced ejection fraction (HFrEF) and heart failure with preserved ejection fraction (HFpEF) and escitalopram in HF reduced ejection fraction (HFrEF) were performed but, results suggested that it did not significantly reduce depression, mortality or rehospitalization $[5,6]$.

Cognitive-behavioral therapy reduced symptoms of depression and improved HF-related quality of life, but 
no data on mortality and readmission was noted [7]. In 2016, the European Society of Cardiology guidelines for diagnosing and treating acute and chronic heart failure included depression as significant comorbidity contributing to the development of HF, especially in the elderly [8]. Since then, depression had become wellestablished comorbidity of HF. However, HFrEF and HFpEF have different etiological profiles and prognoses. In addition, guideline-directed medical therapy (GDMT) with ACEI, ARB, or beta-blockers has proven beneficial in HFrEF, but not HFpEF [9]. Compared with HFrEF, patients with HFpEF are older, more often, women, and more commonly have a history of hypertension and AF [10].

The effect of depression on rehospitalization and mortality between HFrEF and HFpEF is limited to date. We aim to determine the outcome of readmission and mortality in HF patients with and without depression in the current era. We also aimed to identify the prevalence of depression in HF patients using the Nationwide Readmission Database 2018.

\section{Materials And Methods}

We conducted a retrospective cohort study using the National Readmission Database (NRD) for the year 2018. The NRD is a database developed for the Healthcare Cost and Utilization Project (HCUP) sponsored by the Agency for Healthcare Research and Quality through a Federal-State-Industry partnership. In 2018, the NRD contained data from 28 geographically dispersed states, accounting for $\sim 60 \%$ of the total US resident population and $58.7 \%$ of all US hospitalizations [11]. It contains reliable, verifiable patient linkage numbers that could track a patient across hospitals within a state while adhering to strict privacy guidelines. The NRD comprises more than 100 clinical and non-clinical variables for each hospital stay. Each discharge is weighted (weight = total number of discharges from all acute care hospitals in the United States divided by the number of discharges included in the $20 \%$ sample) to calculate the national estimates. NRD has been previously used to provide reliable national readmission estimates in heart failure [12,13]. The NRD 2018 contains patient and hospital-level data with up to 40 diagnoses and 25 procedures collected for each patient using the International Classification of Diseases, Tenth Revision, Clinical Modification (ICD10-CM). We did not obtain institutional review board (IRB) approval due to the data's de-identified nature.

\section{Study population}

We included all patients hospitalized with a primary diagnosis of heart failure. We used appropriate ICD-10 CM codes to identify HFrEF and HFpEF as utilized in the previous study (Table 1) [14]. We identified patients with major depressive disorder (MDD) in the secondary diagnosis field. Patients were excluded if they were $<18$ years old or if the admission was elective. We also excluded patients admitted in December because the NRD captured admission based on a calendar year (i.e., January 1 through December 31) without a link to the previous or following year. We also excluded the readmissions caused by traumatic causes (Figure 1). 


\section{Cureus}

\begin{tabular}{|c|c|}
\hline Variables & ICD-10 codes \\
\hline $\begin{array}{l}\text { Heart failure with a reduced ejection } \\
\text { fraction }\end{array}$ & $1501,1502,1504,15082$ \\
\hline $\begin{array}{l}\text { Heart failure with a preserved ejection } \\
\text { fraction }\end{array}$ & 1503 \\
\hline Major depressive disorder (MDD) & F32, F33 \\
\hline Atrial fibrillation & $1480,14811,14819,14891,1482$ \\
\hline Coronary artery disease & $120,121,122,123,124,125$ \\
\hline Essential hypertension & $110,|11| 12,,|13| 14,,|15| 16$, \\
\hline Diabetes mellitus & E08, E09, E10, E11, E13 \\
\hline Chronic obstructive pulmonary disease & $\mathrm{J} 41, \mathrm{~J} 42, \mathrm{~J} 43, \mathrm{~J} 44$ \\
\hline Obesity & E66, Z683, Z684 \\
\hline Obstructive sleep apnea & G4733 \\
\hline Chronic kidney disease $\geq$ Stage III & N183, N184, N185, E082, E132, I12, I13 \\
\hline End-stage renal disease on hemodialysis & N186, Z992, Z4931, Z4901 \\
\hline Hypothyroidism & E03 \\
\hline Alcohol-related disorders & F10 \\
\hline Anemia & $\begin{array}{l}\text { D50, D51, D52, D53, D55, D56, D57, D58, D59, D60, D61, D62, D63, D64, D460, D461, D462, D464, } \\
\text { O990 }\end{array}$ \\
\hline History of stroke & $1693, \mathrm{Z} 8673$ \\
\hline Peripheral artery disease & E085, E095, E105, E115, E135, I73, T82856, Z9862, Z95820, I252, I2583 \\
\hline Nicotine dependence & F17, Z87891 \\
\hline Cocaine abuse & F14, R782 \\
\hline Schizophrenia & F20 \\
\hline Bipolar disorder & F31 \\
\hline Generalized anxiety disorder & F41 \\
\hline Post-traumatic stress disorder & F431, F432 \\
\hline Attention deficit hyperactivity disorder & F90 \\
\hline
\end{tabular}

\section{TABLE 1: ICD-10 codes used in this study}

ICD-10 = International Classification of Diseases, Tenth Revision 


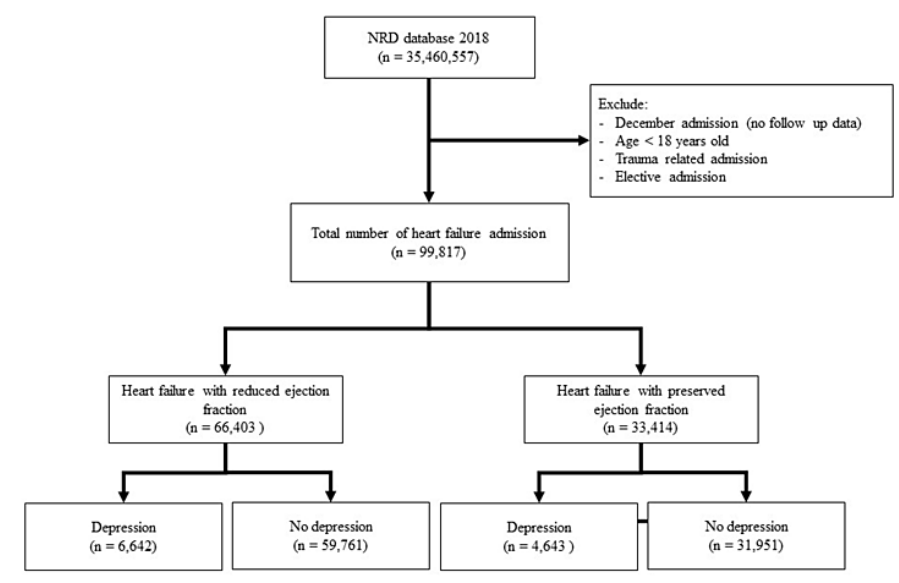

FIGURE 1: Inclusion diagram

NRD = Nationwide Readmissions Database

\section{Study outcomes}

The primary outcome was the composite outcome of all-cause readmission and mortality rate at 30 days for HFrEF with MDD compared to HFrEF without MDD, and then the composite outcome of all-cause readmission and mortality rate at 30 days for $\mathrm{HFpEF}$ with MDD compared to HFpEF without MDD. The secondary outcomes were to (a) compare all-cause readmission rate at 30 days for HFrEF and HFpEF with MDD and without MDD; (b) compare all-cause mortality rate at 30 days for HFrEF and HFpEF with MDD and without MDD; (c) compare resource utilization including total hospitalization charges, cost, and length of stay in HFrEF and HFpEF with MDD and without MDD.

\section{Definition of variables}

We used variables available in the NRD to identify baseline characteristics, including age, gender, median household income for patient's zip code, primary expected payer, admission type, admission day of the week, discharge status, and hospital characteristics (such as bed size and teaching status). The patient comorbidities were identified using the ICD10-CM codes (Table 1). The comorbidity burden was assessed using the Elixhauser Comorbidity Index. Readmission was defined as a non-traumatic admission with any principal diagnosis within 30 days of the index admission. If patients had multiple readmissions within 30 days of discharge, only the first readmission was counted. We used the patient's vital status at discharge for the in-hospital mortality rate, which was directly coded in the database. The three most common reasons for readmission were determined by tallying the principal diagnosis for each readmitted patient. Total hospitalization charges represent the amount that the hospital billed for the entire hospital stay.

\section{Statistical analysis}

The NRD is based on an intricate survey design that includes stratification, clustering, and weighting. The variable "discwt" was used to generate national estimates. Standard error calculations were made considering stratification ("nrd_stratum") and hospitals defining the clusters ("hosp_nrd"). Categorical data were presented as frequency (\%) and were compared using the chi-square test. Continuous data were presented in mean \pm standard deviation (SD) and were compared using the Student's t-test. Unadjusted odds ratio (OR) was calculated by univariate logistic regression for primary and secondary outcomes. Multivariate logistic regression was used to calculate the adjusted OR. Variables that were deemed important determinants of the outcomes based on literature were included in the models. In addition, multiple covariates were built into the model if $\mathrm{p}<0.2$ in univariate analysis. A two-tailed $\mathrm{p}$-value of 0.05 was designated as statistically significant. We adhered to the methodological standard of the Healthcare Cost and Utilization Project (HCUP) [15] and followed the checklist provided by the HCUP [16]. Stata SE, version 16.1 (College Station, Texas: StataCorp.) was used for all statistical analyses.

\section{Results}

From a total of 35,460,557 hospitalizations, 99,817 index admissions of HF were included. Of which, 66,403 admissions were due to HFrEF and 36,594 due to HFpEF. The prevalence of MDD in HFrEF patients was $10 \%$. The prevalence of MDD in HFpEF patients was $13.9 \%$. 


\section{Baseline characteristics}

Complete data of baseline characteristics of HFrEF and HFpEF cohort are presented in Table 2. Most of the HFrEF cohort patients were older than 75 years in the MDD and non-MDD groups. The mean age of HFrEF without MDD was 66.8 years compared to 64.4 years in the MDD group. More females were observed in the MDD cohort (51\% vs $36.4 \%$ ). The most common primary expected payer was Medicare/Medicaid in both groups ( $81.5 \%$ vs $79.6 \%$ ). MDD patients were admitted to teaching hospitals more than the non-MDD group (72.2\% vs $64.9 \%$ ). Non-MDD patients had a higher amount of subjects in the $0-25$ th percentile group than MDD patients (32.8\% vs. $28.8 \%$ ). More significant facilities and home healthcare discharges were noted in the depression group than the non-depression group ( $41.0 \%$ vs $36.2 \%$ ). Patients with MDD had a more significant comorbidity burden with a mean Elixhauser Comorbidity Score (6.3 vs 4.8). The HFrEF with MDD group had higher comorbidity of obesity, OSA, hypertension, diabetes mellitus, prior stroke, end-stage renal disease (ESRD), anemia, hypothyroidism, and anxiety disorder compared to the non-MDD group.

\begin{tabular}{|c|c|c|c|c|c|c|}
\hline \multirow[t]{2}{*}{ Variables } & \multicolumn{3}{|l|}{ HFrEF } & \multicolumn{3}{|l|}{ HFpEF } \\
\hline & $\begin{array}{l}\text { Depression ( } N= \\
6642)\end{array}$ & $\begin{array}{l}\text { No depression }(N= \\
59,761)\end{array}$ & $\begin{array}{l}\mathrm{p}- \\
\text { Value }\end{array}$ & $\begin{array}{l}\text { Depression (N = } \\
4643)\end{array}$ & $\begin{array}{l}\text { No depression }(N= \\
31,951)\end{array}$ & $\begin{array}{l}\mathrm{p}^{\mathrm{p}} \\
\text { Value }\end{array}$ \\
\hline Age (years) (mean) & 64.4 & 66.8 & $<0.01$ & 71.2 & 74.5 & $<0.01$ \\
\hline $18-49$ & $18.6(\%)$ & $15.5(\%)$ & & $7.3(\%)$ & $5.6(\%)$ & \\
\hline $50-64$ & $28.3(\%)$ & $27.1(\%)$ & & $21.9(\%)$ & $17.3(\%)$ & \\
\hline 65-74 & $22.7(\%)$ & $20.3(\%)$ & & $25.2(\%)$ & $19.7(\%)$ & \\
\hline$>75$ & $30.4(\%)$ & $37.0(\%)$ & & $45.5(\%)$ & $57.3(\%)$ & \\
\hline Indicator of gender & & & $<0.01$ & & & $<0.01$ \\
\hline Male & $48.7(\%)$ & $63.6(\%)$ & & $30.2(\%)$ & $41.0(\%)$ & \\
\hline Female & $51.2(\%)$ & $36.4(\%)$ & & $69.8(\%)$ & $58.9(\%)$ & \\
\hline Admission day & & & 0.33 & & & 0.68 \\
\hline Weekday & $76.9(\%)$ & $76.0(\%)$ & & $75.7(\%)$ & $76.0(\%)$ & \\
\hline Weekend & $23.1(\%)$ & $24.0(\%)$ & & $24.3(\%)$ & $23.9(\%)$ & \\
\hline Primary insurance & & & $<0.01$ & & & 0.22 \\
\hline Federal/state $^{\mathrm{a}}$ & $81.5(\%)$ & $79.6(\%)$ & & $89.3(\%)$ & $88.2(\%)$ & \\
\hline Private & $16.2(\%)$ & $15.6(\%)$ & & $9.5(\%)$ & $10.3(\%)$ & \\
\hline Uninsured & $2.2(\%)$ & $4.7(\%)$ & & $1.1(\%)$ & $1.4(\%)$ & \\
\hline Hospital bed size ${ }^{b}$ & & & 0.06 & & & 0.11 \\
\hline Small/Medium & $44.1(\%)$ & $46.6(\%)$ & & $50.7(\%)$ & $53.0(\%)$ & \\
\hline Large & $55.9(\%)$ & $53.4(\%)$ & & $49.2(\%)$ & $46.9(\%)$ & \\
\hline Hospital teaching status $^{c}$ & & & $<0.01$ & & & 0.03 \\
\hline Non-teaching & $27.8(\%)$ & $35.1(\%)$ & & $37.2(\%)$ & $40.2(\%)$ & \\
\hline Teaching & $72.2(\%)$ & $64.9(\%)$ & & $62.8(\%)$ & $59.8(\%)$ & \\
\hline $\begin{array}{l}\text { Median household income by zip-code, } \\
\text { percentile }^{d}\end{array}$ & & & $<0.01$ & & & 0.03 \\
\hline $0-25$ th & $28.8(\%)$ & $32.8(\%)$ & & $25.2(\%)$ & $26.3(\%)$ & \\
\hline 26-50th & $29.1(\%)$ & $29.7(\%)$ & & $29.7(\%)$ & $32.2(\%)$ & \\
\hline 51-75th & $24.7(\%)$ & $22.8(\%)$ & & $26.4(\%)$ & $24.3(\%)$ & \\
\hline 76-100th & $17.4(\%)$ & $14.7(\%)$ & & $18.5(\%)$ & $17.1(\%)$ & \\
\hline Disposition & & & $<0.01$ & & & $<0.01$ \\
\hline Home/self-care & $55.3(\%)$ & $59.2(\%)$ & & $42.5(\%)$ & $46.8(\%)$ & \\
\hline
\end{tabular}




\section{Cureus}

\begin{tabular}{|c|c|c|c|c|c|c|}
\hline Short-term hospital & $1.6(\%)$ & $1.9(\%)$ & & $1.1(\%)$ & $1.1(\%)$ & \\
\hline Facilitye & $16.9(\%)$ & $14.1(\%)$ & & $26.5(\%)$ & $23.5(\%)$ & \\
\hline Home health care & $24.1(\%)$ & $22.1(\%)$ & & $28.7(\%)$ & $27.4(\%)$ & \\
\hline Against medical advice & $1.9(\%)$ & $2.6(\%)$ & & $1.0(\%)$ & $1.0(\%)$ & \\
\hline Elixhauser Comorbidity Index Score & & & $<0.01$ & & & $<0.01$ \\
\hline 1 & $0(\%)$ & 2.1 & & 0 & 0.9 & \\
\hline 2 & $1.1(\%)$ & 7.8 & & 0.7 & 5.1 & \\
\hline 3 & $5.4(\%)$ & 15.4 & & 3.4 & 12.3 & \\
\hline$>4$ & $93.4(\%)$ & 74.6 & & 95.8 & 81.5 & \\
\hline Mean Elixhauser Comorbidity Score & 6.3 & 4.8 & $<0.01$ & 6.4 & 5.2 & $<0.01$ \\
\hline \multicolumn{7}{|l|}{ Comorbidities $^{f}$} \\
\hline Atrial fibrillation & $37.3(\%)$ & $37.6(\%)$ & 0.74 & $43.9(\%)$ & $48.6(\%)$ & $<0.01$ \\
\hline Obesity & $24.3(\%)$ & $17.1(\%)$ & $<0.01$ & $35.8(\%)$ & $26.2(\%)$ & $<0.01$ \\
\hline OSA & $19.2(\%)$ & $10.5(\%)$ & $<0.01$ & $24.2(\%)$ & $16.3(\%)$ & $<0.01$ \\
\hline Hypertension & $34.0(\%)$ & $31.3(\%)$ & 0.01 & $38.6(\%)$ & $38.1(\%)$ & 0.67 \\
\hline Diabetes mellitus & $35.2(\%)$ & $31.5(\%)$ & $<0.01$ & $38.5(\%)$ & $34.2(\%)$ & $<0.01$ \\
\hline CAD & $51.3(\%)$ & $51.5(\%)$ & 0.85 & $35.8(\%)$ & $37.4(\%)$ & 0.17 \\
\hline Prior stroke & $11.2(\%)$ & $8.5(\%)$ & $<0.01$ & $11.9(\%)$ & $9.9(\%)$ & $<0.01$ \\
\hline CKD stage $>3$ & $25.9(\%)$ & $24.6(\%)$ & 0.16 & $24.7(\%)$ & $26.2(\%)$ & 0.15 \\
\hline ESRD & $2.7(\%)$ & $2.0(\%)$ & 0.02 & $2.1(\%)$ & $2.6(\%)$ & 0.22 \\
\hline Peripheral vascular disease & $19.9(\%)$ & $19.0(\%)$ & 0.23 & $14.8(\%)$ & $13.1(\%)$ & 0.03 \\
\hline Anemia & $28.8(\%)$ & $24.3(\%)$ & $<0.01$ & $33.8(\%)$ & $31.3(\%)$ & 0.01 \\
\hline Hypothyroidism & $19.5(\%)$ & $13.2(\%)$ & $<0.01$ & $24.5(\%)$ & $19.9(\%)$ & $<0.01$ \\
\hline Alcohol & $6.5(\%)$ & $6.8(\%)$ & 0.51 & $3.4(\%)$ & $2.8(\%)$ & 0.18 \\
\hline Smoking & $50.3(\%)$ & $46.2(\%)$ & $<0.01$ & $45.1(\%)$ & $39.3(\%)$ & $<0.01$ \\
\hline Cocaine use & $2.4(\%)$ & $2.4(\%)$ & 0.82 & $0.6(\%)$ & $0.5(\%)$ & 0.60 \\
\hline Schizophrenia & $1.3(\%)$ & $1.0(\%)$ & 0.09 & $1.0(\%)$ & $0.8(\%)$ & 0.27 \\
\hline Bipolar disorder & $0.3(\%)$ & $1.5(\%)$ & $<0.01$ & $0.4(\%)$ & $1.7(\%)$ & $<0.01$ \\
\hline Anxiety disorder & $34.9(\%)$ & $8.4(\%)$ & $<0.01$ & $35.3(\%)$ & $9.2(\%)$ & $<0.01$ \\
\hline PTSD/adjustment disorder & $3.4(\%)$ & $0.9(\%)$ & $<0.01$ & $2.2(\%)$ & $0.7(\%)$ & $<0.01$ \\
\hline ADHD & $1.9(\%)$ & $0.3(\%)$ & $<0.01$ & $0.4(\%)$ & $0.1(\%)$ & $<0.01$ \\
\hline
\end{tabular}

TABLE 2: Baseline characteristics for heart failure patients with and without depression: stratified by ejection fraction, National Readmission Database, 2018

$\mathrm{ADHD}=$ attention-deficit/hyperactivity disorder; $\mathrm{CAD}=$ coronary artery disease; $\mathrm{CKD}=$ chronic kidney disease; ESRD = end-stage renal disease; $\mathrm{HFpEF}$ = heart failure preserved ejection fraction; HFrEF = heart failure reduced ejection fraction; $\mathrm{PTSD}=$ post-traumatic stress disorder; OSA = obstructive sleep apnea; no. = number

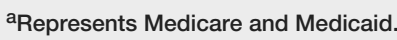

bSmall bed-size hospitals contain 1-250 beds, medium bed-size contains 50-450 beds and large bed-size hospitals contain 450+ beds.

${ }^{\mathrm{c}} \mathrm{A}$ hospital is considered a teaching hospital if it has one or more Accreditation Council for Graduate Medical Education (ACGME) approved residency program, is a member of the Council of Teaching Hospitals (COTH), or has a ratio of full-time equivalent interns and residents to beds of 0.25 or higher (https://www.hcup-us.ahrq.gov/db/vars/hosp_bedsize/nrdnote.jsp).

${ }^{\mathrm{d}}$ Represents a quartile classification of the estimated median household income of residents in the patient's ZIP Code. The quartiles are identified 


\section{Cureus}

by values of one to four, indicating the poorest to wealthiest populations. Because these estimates are updated annually, the values of one to four vary by year (https://www.hcup-us.ahrq.gov/db/vars/zipinc_qrt//nrdnote.jsp).

eDisposition to skilled nursing facility (SNF), intermediate care facility (ICF), Hospice - medical facility, long-term care hospital swing bed, rehabilitation facility, nursing facility certified by Medicaid or transfer to a psychiatric hospital (https://www.hcup-

us.ahrq.gov/db/vars/dispuniform/nrdnote.jsp).

fICD-10 codes were utilized to identify respective comorbidity as per Table 1.

In the HFpEF cohort, patients without MDD were older, with a mean age of 74.5 years compared to 71.2 years. Female patients were more prevalent in the MDD group than without depression (69.8\% vs 58.9\%). HFrEF with and without MDD had non-significant differences in day of admission, primary insurance, and hospital bed size. Facility discharge and discharges with home healthcare were higher in the MDD group (55.2\% vs 50.9\%: $\mathrm{p}<0.01$ ). HFpEF patients with MDD had a more significant comorbidity burden with a mean Elixhauser comorbidity score (6.4 vs 5.2: $\mathrm{p}<0.01$ ). Hospitalizations with MDD had higher comorbidity of obesity, OSA, diabetes mellitus, hypothyroidism, smoking, and anxiety.

\section{Primary outcome: composite of all-cause readmissions and mortality in 30 days}

In the HFrEF cohort, a total of 6642 indexes HFrEF with MDD hospitalizations were discharged alive. The composite outcome of all-cause readmission and mortality at 30 days occurred in $20.1 \% \mathrm{HFrEF}$ patients with MDD compared to 20.0\% in patients without MDD. Adjusted OR was 0.94 (95\% CI: 0.85-1.04) (Table 3).

\begin{tabular}{|c|c|c|c|c|c|c|}
\hline \multicolumn{7}{|c|}{ Heart failure with reduced ejection fraction } \\
\hline Outcome & $\begin{array}{l}\text { Depression }(n= \\
6642)\end{array}$ & $\begin{array}{l}\text { No depression (n } \\
=59,761)\end{array}$ & $\begin{array}{l}\text { Unadjusted odds ratio } \\
\text { a }\end{array}$ & $\begin{array}{l}\mathrm{p}- \\
\text { Value }\end{array}$ & $\begin{array}{l}\text { Adjusted odd } \\
\text { ratio }^{a, b}\end{array}$ & $\begin{array}{l}\mathrm{p}- \\
\text { Value }\end{array}$ \\
\hline Primary outcome $\mathrm{C}^{\mathrm{C}}$ & $1335(20.1 \%)$ & 11996 (20.0\%) & $1.00(0.92-1.09)$ & 0.97 & $0.94(0.85-1.04)$ & 0.27 \\
\hline $\begin{array}{l}30 \text { day all-cause } \\
\text { readmission }\end{array}$ & $1146(17 \%)$ & $9759(16.3 \%)$ & $1.07(0.97-1.17)$ & 0.16 & $0.94(0.85-1.05)$ & 0.27 \\
\hline 30-day mortality & $241(3.6 \%)$ & $2778(4.8 \%)$ & $0.75(0.60-0.94)$ & 0.01 & $0.79(0.62-1.00)$ & 0.05 \\
\hline \multicolumn{7}{|c|}{ Heart failure with preserved ejection fraction } \\
\hline Outcome & $\begin{array}{l}\text { Depression }(n= \\
4643)\end{array}$ & $\begin{array}{l}\text { No depression }(n \\
=31,951)\end{array}$ & $\begin{array}{l}\text { Unadjusted odds } \\
\text { ratio }^{\mathrm{a}}\end{array}$ & $\begin{array}{l}\mathrm{p}- \\
\text { Value }\end{array}$ & $\begin{array}{l}\text { Adjusted odd } \\
\text { ratio }^{\mathrm{a}, \mathrm{b}}\end{array}$ & $\begin{array}{l}\mathrm{p}- \\
\text { Value }\end{array}$ \\
\hline Primary outcome $\mathrm{C}^{\mathrm{C}}$ & $861(18.5 \%)$ & $5928(18.5 \%)$ & $1.00(0.88-1.12)$ & 0.99 & $0.93(0.81-1.07)$ & 0.32 \\
\hline $\begin{array}{l}\text { 30-day all-cause } \\
\text { readmission }\end{array}$ & 774 (16.6\%) & 4942 (15.4\%) & $1.09(0.96-1.23)$ & 0.15 & $0.93(0.81-1.07)$ & 0.32 \\
\hline 30-day mortality & $87(1.9 \%)$ & 985 (3.0\%) & $0.60(0.44-0.82)$ & $<0.01$ & $0.69(0.50-0.95)$ & 0.02 \\
\hline
\end{tabular}

\section{TABLE 3: Summary of outcomes of the study including rate and odds ratio of HFrEF and HFpEF with and without major depressive disorder}

$\mathrm{HFpEF}=$ heart failure with preserved ejection fraction; $\mathrm{HFrEF}=$ heart failure with reduced ejection fraction

aOdds ratio for heart failure with depression compared to heart failure without depression.

${ }^{b}$ Data were adjusted for age, gender, hospital bed size, hospital teaching status, median household income, disposition, Elixhauser comorbidity index, atrial fibrillation, obesity, obstructive sleep apnea, hypertension, diabetes mellitus, coronary artery disease, prior stroke, chronic kidney disease, anemia, hypothyroidism, smoker, schizophrenia, bipolar disorder, anxiety disorder, post-traumatic stress disorder/adjustment disorder, and attention-deficit/hyperactivity disorder.

${ }^{\mathrm{C}}$ Primary outcome consisted of combined 30-day all-cause readmission and mortality.

For the HFpEF cohort, a total of 4643 indexes HFpEF with MDD hospitalizations were discharged alive. The composite outcome of all-cause readmission and mortality at 30 days occurred in $18.5 \%$ on both HFpEF with and without MDD. Adjusted odds ratio (OR) was 0.93 (95\% CI: 0.81-1.07). Lower median household income, leaving against medical advice, and higher Elixhauser Comorbidity Index Score were identified as major 


\section{Cureus}

predictors of readmission and mortality in HFrEF and HFpEF groups (Table 4).

\begin{tabular}{|c|c|c|c|c|}
\hline \multirow{2}{*}{ Variables } & \multicolumn{2}{|l|}{ HFrEF } & \multirow{2}{*}{$\begin{array}{l}\text { HFpEF } \\
\text { Unadjusted odds ratio }\end{array}$} & \multirow[b]{2}{*}{ p-Value } \\
\hline & Unadjusted odds ratio & p-Value & & \\
\hline Age & 1.0 & 0.56 & 0.99 & 0.32 \\
\hline \multicolumn{5}{|l|}{ Gender } \\
\hline Male & \multicolumn{2}{|l|}{ Reference } & \multicolumn{2}{|l|}{ Reference } \\
\hline Female & 0.9 & $<0.01$ & 1.00 & 0.81 \\
\hline Admission day & 1.05 & 0.11 & 1.08 & 0.06 \\
\hline \multicolumn{5}{|l|}{ Primary insurance } \\
\hline Federal/state & \multicolumn{2}{|l|}{ Reference } & \multicolumn{2}{|l|}{ Reference } \\
\hline Private & 0.68 & $<0.01$ & 0.77 & $<0.01$ \\
\hline Uninsured & 0.76 & $<0.01$ & 0.94 & 0.75 \\
\hline \multicolumn{5}{|l|}{ Hospital bed size } \\
\hline Small/medium & \multicolumn{2}{|l|}{ Reference } & \multicolumn{2}{|l|}{ Reference } \\
\hline Large & 1.00 & 0.80 & 1.12 & $<0.01$ \\
\hline \multicolumn{5}{|l|}{ Hospital teaching status } \\
\hline Non-teaching & Reference & & Reference & \\
\hline Teaching & 0.93 & 0.05 & 0.94 & 0.22 \\
\hline \multicolumn{5}{|c|}{ Median household income category for patient's zip-code } \\
\hline $0-25$ th & Reference & & Reference & \\
\hline 26-50th & 0.83 & $<0.01$ & 0.92 & 0.11 \\
\hline 51-75th & 0.85 & $<0.01$ & 0.89 & 0.04 \\
\hline 76-100th & 0.78 & $<0.01$ & 0.80 & $<0.01$ \\
\hline \multicolumn{5}{|l|}{ Disposition } \\
\hline Home & Reference & & Reference & \\
\hline Short-term hospital & 1.19 & 0.11 & 1.84 & $<0.01$ \\
\hline Facility & 1.04 & 0.38 & 1.15 & $<0.01$ \\
\hline Home health care & 1.11 & $<0.01$ & 1.19 & $<0.01$ \\
\hline Against medical advice & 2.76 & $<0.01$ & 1.94 & $<0.01$ \\
\hline \multicolumn{5}{|c|}{ Elixhauser Comorbidity Index Score } \\
\hline 1 & Reference & & Reference & \\
\hline 2 & 0.85 & 0.27 & 1.16 & 0.58 \\
\hline 3 & 1.08 & 0.56 & 1.31 & 0.30 \\
\hline$>4$ & 1.45 & $<0.01$ & 1.99 & $<0.01$ \\
\hline \multicolumn{5}{|l|}{ Co-morbidities } \\
\hline Atrial fibrillation & 1.08 & $<0.01$ & 1.18 & $<0.01$ \\
\hline Obesity & 0.88 & $<0.01$ & 0.92 & 0.06 \\
\hline OSA & 0.91 & 0.05 & 1.00 & 0.95 \\
\hline Hypertension & 1.10 & $<0.01$ & 1.07 & 0.09 \\
\hline
\end{tabular}




\section{Cureus}

\begin{tabular}{lllll|} 
Diabetes mellitus & 1.14 & $<0.01$ & 1.13 & $<0.01$ \\
CAD & 1.18 & $<0.01$ & 1.18 & $<0.01$ \\
Prior stroke & 1.16 & $<0.01$ & 1.14 & 0.04 \\
CKD stage $>3$ & 1.36 & $<0.01$ & 1.40 & $<0.01$ \\
ESRD & 1.81 & $<0.01$ & 1.54 & $<0.01$ \\
Peripheral vascular disease & 1.20 & $<0.01$ & 1.13 & 0.02 \\
Anemia & 1.31 & $<0.01$ & 1.24 & $<0.01$ \\
Hypothyroidism & 1.13 & $<0.01$ & 1.09 & 0.05 \\
Alcohol & 0.92 & 0.18 & 0.95 & 0.68 \\
Smoking & 0.91 & $<0.01$ & 0.97 & 0.42 \\
Cocaine use & 1.30 & $<0.01$ & 1.49 & 0.08 \\
Schizophrenia & 1.55 & $<0.01$ & 0.86 & 0.54 \\
Bipolar disorder & 1.06 & 0.58 & 1.21 & 0.16 \\
Anxiety disorder & 1.15 & $<0.01$ & 1.05 & 0.40 \\
PTSD/adjustment disorder & 1.10 & 0.42 & 0.77 & 0.20 \\
ADHD & 0.74 & 0.21 & 1.03 & 0.94 \\
\hline
\end{tabular}

TABLE 4: Unadjusted predictors of combined 30-day all-cause readmission and mortality for each baseline characteristics

$\mathrm{ADHD}=$ attention-deficit/hyperactivity disorder; $\mathrm{CAD}=$ coronary artery disease; $\mathrm{CKD}=$ chronic kidney disease; $\mathrm{ESRD}=\mathrm{end}$-stage renal disease; $\mathrm{HFpEF}=$ heart failure preserved ejection fraction; HFrEF = heart failure reduced ejection fraction; $\mathrm{PTSD}=$ post-traumatic stress disorder; OSA = obstructive sleep apnea

\section{Secondary outcomes}

All-Cause Readmission Rate at 30 Days

In the HFrEF cohort, $17 \%$ of patients with MDD were readmitted within 30 days. The three most common principal diagnoses for readmissions in the MDD cohort were acute heart failure (44.9\%), sepsis (4.5\%), and acute kidney failure (2.9\%). HFrEF without MDD had a $16.3 \%$ rate of readmission. Comparing HFrEF patients with MDD and without MDD, the adjusted OR was 0.94 (95\% CI: 0.85-1.05) (Table 3).

In the HFpEF cohort, the 30-day all-cause readmission rate for patients with MDD was $16.6 \%$ compared to $15.4 \%$ in the non-MDD group. The three most common principal diagnoses for readmissions in HFpEF with MDD were acute heart failure (41.0\%), sepsis (7.2\%), and acute and chronic hypoxic respiratory failure (4.9\%). Adjusted OR was 0.93 (95\% CI: 0.81 - 1.07) when compared HFpEF with MDD to without MDD.

\section{All-Cause Mortality Rate at 30 Days}

In the HFrEF cohort, the 30-day mortality rate in the MDD and non-MDD groups was $2.8 \%$ and $3.6 \%$, respectively. MDD was associated with lower mortality than non-MDD in HFrEF with an adjusted OR of 0.79 (95\% CI: 0.62-1.00). In the HFpEF cohort, the 30-day mortality rate in the MDD group was $1.9 \%$ and $3 \%$ in the non-MDD group. HFpEF patients with MDD had lower mortality than those without MDD, with an adjusted OR of 0.69 (95\% CI: 0.5-0.95).

\section{Resource utilization}

In the HFrEF cohort, patients with MDD had a longer mean length of stay (6.7 vs 5.7 days; $p<0.01$ ) (Table 5). HFrEF with MDD had higher mean total initial hospitalization charges compared to those without MDD (USD $\$ 69,810$ vs $\$ 62,935 ; p=0.03)$. Readmission cost was higher in both HFrEF with and without MDD compared to the index admission. 


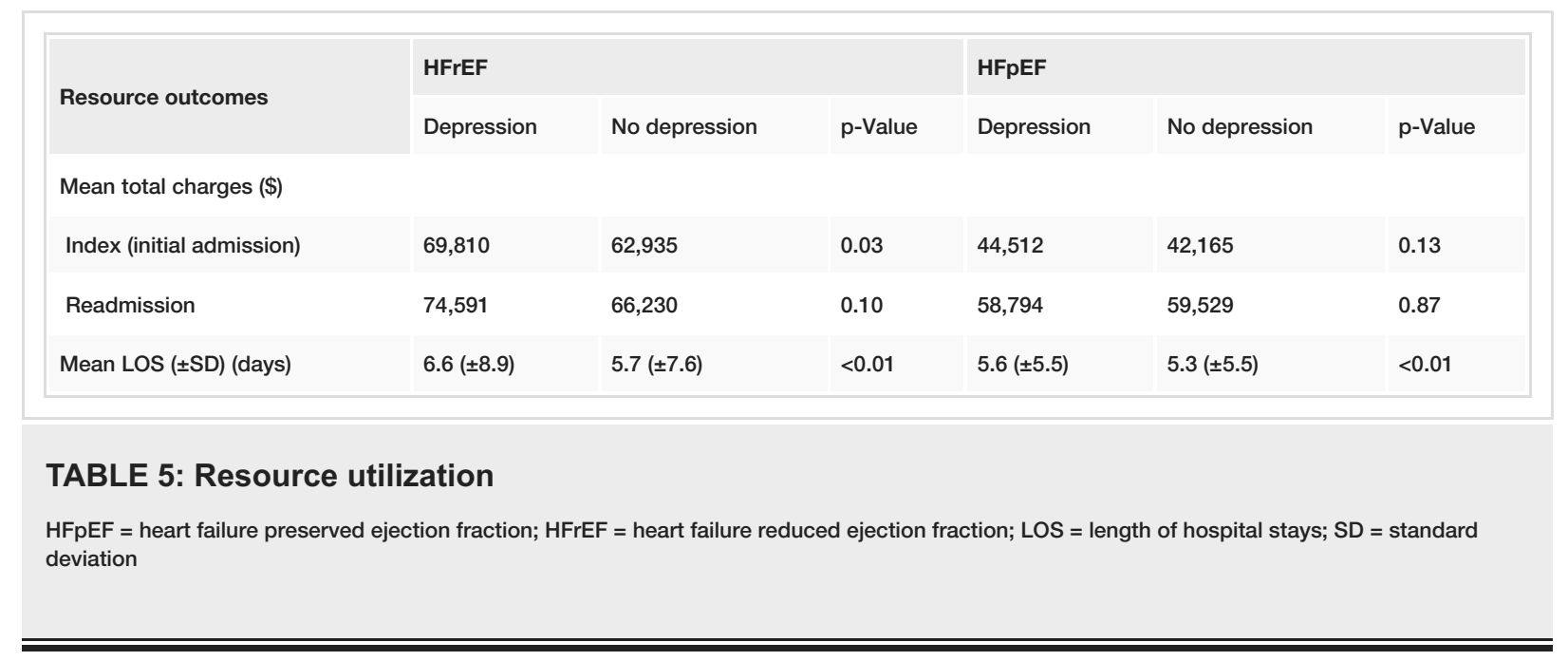

In the HFpEF cohort, patients with MDD had a longer length of stay than the non-MDD group ( 5.6 vs 5.3 days; $p<0.01$ ). The mean total hospitalization charges were not statistically different between MDD and non-MDD groups ( $\$ 44,512$ vs $\$ 42,165 ; \mathrm{p}=0.13)$.

\section{Discussion}

This is the first study investigating the association of MDD and HF in terms of HFrEF and HFpEF. Using NRD 2018, a large US national database, we found a total prevalence of $11 \%$ for all types of HF patients with MDD. Patients with HFrEF had less prevalence of MDD (10\%) compared to HFpEF (13.9\%). The combined rate of all-cause readmission and mortality at 30 days was not statistically different in both types of HF patients (HFrEF and HFpEF) with or without MDD. Both types of heart failure with concomitant depression were not associated with all-cause 30-day readmissions but were associated with decreased mortality rates in both groups.

Heart failure patients with MDD were younger and more likely to be female than the group without MDD. Patients in the heart failure with preserved ejection fraction group were older than HFrEF with an average age of 72-75 years compared to 64-67 years. HFrEF with MDD had more comorbidity of obesity, OSA, hypothyroidism, tobacco use, anemia, and a higher Elixhauser Comorbidity Index Score than those without MDD. This pattern was similar to HFpEF with MDD, except that the HFpEF without MDD group had a higher prevalence of atrial fibrillation. These comorbidities were associated with increased HF mortality and rehospitalization, except for obesity [17-20]. Obesity is highly prevalent in HF groups and interestingly associated with lower mortality in HF patients [18]. In our study, obesity was more commonly associated with the MDD group, which could have contributed to lesser mortality in the MDD group for both types of HF. In our study, underlying diseases involving psychiatric issues, such as anxiety disorder and PTSD, were more prevalent in HF with MDD than patients without MDD. Lin et al., had shown that anxiety was not associated with readmission or mortality at an 18-month duration in the HFrEF group but had increased readmission and mortality in the HFpEF group [21]. Similarly, in our study, anxiety disorder was more prevalent in MDD groups and did not result in a higher readmission rate. However, we noticed lower mortality in MDD groups. This difference could be related to the shorter 30-day follow-up period in our study. Patients with HFrEF with MDD were less likely to be discharged to home compared to those without MDD. On the contrary, HFpEF patients with MDD were likely to discharge home compared to those without MDD. These comorbidities affected outcomes differently across all HF patients, both with and without MDD. Therefore, we used multivariate regression analysis to adjust for all the comorbidities to find the actual impact of MDD on HF.

We found that MDD was not associated with an all-cause readmission rate for both types of HF. This finding was different from a previous NRD study that used the 2010-2014 database which showed an increased readmission rate in the MDD group compared to non-MDD [2]. This difference could be related to the study's inclusion of all types of heart failure but not specific HFrEF and HFpEF ICD codes. The current expert recommendations for depression in HF patients are similar to non-HF patients however, antidepressant efficacy has not been demonstrated yet in the HF patients but the more effective therapies are psychotherapy and care management [22]. This is unfortunately not measurable in our study due to the inherent limitation of the database itself. In our study, HFrEF and HFpEF with MDD were associated with similar readmission rates and significantly lower mortality at 30 days compared to those without MDD. This finding is different from the previous studies that showed increased mortality in HF patients with depression of two to four-fold $[1,2]$. In theory, HF patients with MDD could have received more attention, follow-ups, and multidisciplinary care compared to HF patients without MDD. This could contribute to reduced mortality. Preliminary results of the Blended Collaborative Care for Heart Failure and Co-morbid depression trial (NCT02044211) in HFrEF patients with depression showed that collaborative care of psychiatrists, cardiologists, internists, and nurses, reduced patients mood symptoms but not mortality compared to HF 
patients without depression [23]. Unfortunately, NRD does not contain the medications, psychotherapy, and social support data that could influence a positive outcome. More research is needed to pinpoint the most effective treatment for HF patients with depression.

\section{Future perspective}

Prospective studies are needed to strengthen the association and establish causality for decreased mortality with patients with MDD compared to HF patients without MDD. Better screening, treatment, and management of depression could reduce the worsening outcomes in HF patients. Studies with more extended follow-up periods are needed to assess long-term readmission differences in this patient population.

\section{Limitations}

We acknowledge several limitations in this study. First, NRD relies on administrative coding, and data depends on the rigor of the institutions involved. Second, NRD does not contain information on medications, laboratory values, echocardiographic data, and other treatment modalities like cognitive behavioral therapy, limiting our assessment of the studied association. Third, the database does not account for deaths outside the hospital, impacting the mortality association. Despite these limitations, this large population study contains half of the US rehospitalized patient population and may provide significant external validity and generalizability findings.

\section{Conclusions}

The prevalence of MDD in HFpEF was higher than HFrEF at $13.9 \%$ and 10\%, respectively. Concomitant MDD in HF patients was not associated with the combined outcome of in-hospital mortality and rehospitalization at 30 days. However, the secondary outcome of 30-day mortality was significantly lower in HFrEF and HFpEF patients with MDD compared to patients without MDD. There are several inherent limitations of the NRD database which limits us from examining the factors contributing to the decreased mortality in the MDD group. Further studies are needed with a longer follow-up period and increased granularity of the data to verify the results of this study and also to determine the treatment that influences readmission and mortality in HF patients with MDD.

\section{Additional Information \\ Disclosures}

Human subjects: Consent was obtained or waived by all participants in this study. Animal subjects: All authors have confirmed that this study did not involve animal subjects or tissue. Conflicts of interest: In compliance with the ICMJE uniform disclosure form, all authors declare the following: Payment/services info: All authors have declared that no financial support was received from any organization for the submitted work. Financial relationships: All authors have declared that they have no financial relationships at present or within the previous three years with any organizations that might have an interest in the submitted work. Other relationships: All authors have declared that there are no other relationships or activities that could appear to have influenced the submitted work.

\section{Acknowledgements}

The authors would like to thank Matthew Roslund, Bassett Healthcare Network's librarian, for proofreading the manuscript and help with the extensive literature search.

\section{References}

1. Rutledge T, Reis VA, Linke SE, Greenberg BH, Mills PJ: Depression in heart failure a meta-analytic review of prevalence, intervention effects, and associations with clinical outcomes. J Am Coll Cardiol. 2006, 48:15271537. 10.1016/j.jacc.2006.06.055

2. Moraska AR, Chamberlain AM, Shah ND, et al.: Depression, healthcare utilization, and death in heart failure: a community study. Circ Heart Fail. 2013, 6:387-394. 10.1161/CIRCHEARTFAILURE.112.000118

3. Patel N, Chakraborty S, Bandyopadhyay D, et al.: Association between depression and readmission of heart failure: a national representative database study. Prog Cardiovasc Dis. 2020, 63:585-590. 10.1016/i.pcad.2020.03.014

4. Adelborg K, Schmidt M, Sundbøll J, et al.: Mortality risk among heart failure patients with depression: a nationwide population-based cohort study. J Am Heart Assoc. 2016, 5: 10.1161/JAHA.116.004137

5. O'Connor CM, Jiang W, Kuchibhatla M, et al.: Safety and efficacy of sertraline for depression in patients with heart failure: results of the SADHART-CHF (sertraline against depression and heart disease in chronic heart failure) trial. J Am Coll Cardiol. 2010, 56:692-699. 10.1016/j.jacc.2010.03.068

6. Angermann CE, Gelbrich G, Störk S, et al.: Effect of escitalopram on all-cause mortality and hospitalization in patients with heart failure and depression: the MOOD-HF randomized clinical trial. JAMA. 2016, 315:2683-2693. 10.1001/jama.2016.7635

7. Freedland KE, Carney RM, Rich MW, Steinmeyer BC, Rubin EH: Cognitive behavior therapy for depression and self-care in heart failure patients a randomized clinical trial. JAMA Intern Med. 2015, 175:1773-1782.

10.1001/jamainternmed.2015.5220 
8. Ponikowski P, Voors AA, Anker SD, et al.: 2016 ESC Guidelines for the diagnosis and treatment of acute and chronic heart failure: the task force for the diagnosis and treatment of acute and chronic heart failure of the European Society of Cardiology (ESC). Developed with the special contribution of the Heart Failure Association (HFA) of the ESC. Eur Heart J. 2016, 37:2129-2200. 10.1093/eurheartj/ehw128

9. Borlaug BA: Evaluation and management of heart failure with preserved ejection fraction . Nat Rev Cardiol. 2020, 17:559-573. 10.1038/s41569-020-0363-2

10. Dunlay SM, Roger VL, Redfield MM: Epidemiology of heart failure with preserved ejection fraction . Nat Rev Cardiol. 2017, 14:591-602. 10.1038/nrcardio.2017.65

11. Overview of the Nationwide Readmissions Database (NRD) . (2021). Accessed: April 28, 2021: https://www.hcup-us.ahrq.gov/nrdoverview.jsp.

12. Nizamuddin J, Gupta A, Patel V, et al.: Hypertensive diseases of pregnancy increase risk of readmission with heart failure: a National Readmissions Database Study. Mayo Clin Proc. 2019, 94:811-819. 10.1016/j.mayocp.2018.08.032

13. Ram P, Shah M, Lo KBU, et al.: Etiologies and predictors of readmission among obese and morbidly obese patients admitted with heart failure. [In Press]. Heart Fail Rev. 2020, 10.1007/s10741-020-09920-4

14. Arora S, Jaswaney R, Jani C, et al.: Catheter ablation for atrial fibrillation in patients with concurrent heart failure. Am J Cardiol. 2020, 137:45-54. 10.1016/j.amjcard.2020.09.035

15. Khera R, Angraal S, Couch T, et al.: Adherence to methodological standards in research using the National Inpatient Sample. JAMA. 2017, 318:2011-2018. 10.1001/jama.2017.17653

16. Checklist for working with the NRD . (2020). Accessed: April 28, 2021: https://www.hcupus.ahrq.gov/db/nation/nrd/nrdchecklist.jsp.

17. Wang H, Parker JD, Newton GE, et al.: Influence of obstructive sleep apnea on mortality in patients with heart failure. J Am Coll Cardiol. 2007, 49:1625-1631. 10.1016/j.jacc.2006.12.046

18. Ning N, Gao D, Triggiani V, et al.: Prognostic role of hypothyroidism in heart failure: a meta-analysis . Medicine (Baltimore). 2015, 94:e1159. 10.1097/MD.0000000000001159

19. Mozaffarian D, Nye R, Levy WC: Anemia predicts mortality in severe heart failure: the prospective randomized amlodipine survival evaluation (PRAISE). J Am Coll Cardiol. 2003, 41:1933-1939. 10.1016/S0735-1097(03)00425-X

20. Gustafsson F, Kragelund CB, Torp-Pedersen C, et al.: Effect of obesity and being overweight on long-term mortality in congestive heart failure: influence of left ventricular systolic function. Eur Heart J. 2005, 26:5864. 10.1093/eurheartj/ehi022

21. Lin TK, Hsu BC, Li YD, Chen CH, Lin JW, Chien CY, Weng CY: Prognostic value of anxiety between heart failure with reduced ejection fraction and heart failure with preserved ejection fraction. J Am Heart Assoc. 2019, 8:e010739. 10.1161/JAHA.118.010739

22. Zambrano J, Celano CM, Januzzi JL, Massey CN, Chung WJ, Millstein RA, Huffman JC: Psychiatric and psychological interventions for depression in patients with heart disease: a scoping review. J Am Heart Assoc. 2020, 9:e018686. 10.1161/JAHA.120.018686

23. Herbeck Belnap B, Anderson A, Abebe KZ, Ramani R, Muldoon MF, Karp JF, Rollman BL: Blended collaborative care to treat heart failure and comorbid depression: rationale and study design of the hopeful heart trial. Psychosom Med. 2019, 81:495-505. 10.1097/PSY.0000000000000706 